\title{
A Psychophysiological Examination of the Mutability of Type D Personality in a Therapeutic Trial
}

\author{
Kirsty L. Hodgson ${ }^{1}$, Debbie A. Clayton ${ }^{1}$, Michael A. Carmi ${ }^{2}$, Louise H. Carmi ${ }^{2}$, \\ Ronald A. Ruden ${ }^{3}$, William D. Fraser ${ }^{4}$, and Donnie Cameron ${ }^{5}$ \\ ${ }^{1}$ Department of Applied Psychology, Cardiff Metropolitan University, Cardiff, UK \\ ${ }^{2}$ Psychological Therapies, Harley Street Therapy Centre, London, UK \\ ${ }^{3}$ Yaffe Ruden and Associates, Primary Care, New York, NY, USA \\ ${ }^{4}$ Department of Endocrinology and Department of Clinical Biochemistry, Norfolk and Norwich University Hospital, \\ University of East Anglia, Norwich, UK \\ ${ }^{5}$ Norwich Medical School, University of East Anglia, Norwich, UK
}

\begin{abstract}
Identifying the associations between health and personality has been a focus for psychophysiological research. Type D personality is associated with predisposition to physical and psychological ill-health. This statistician-blind parallel-group controlled trial (intervention group vs. waiting list control group) examined the impact of Havening Techniques on the Type D constituents of negative affect (NA) and social inhibition (SI). One hundred twenty-five adult (18+ years) participants in the United Kingdom (72 females, 53 males) completed the Type D Scale-14 (DS14) measure of Type D personality at baseline (T1), 24-hours (T2), and at 1-month (T3). Forty participants in the treatment group received additional stress biomarker assessment of heart rate, blood pressure, and salivary cortisol. Type $D$ caseness remained stable in the waiting list participants $(n=57)$. In the treatment group $(n=68)$; NA, SI, and total scores decreased from T1 to T2 $(p<.001, p<.001$, and $p<.001$, respectively), and from T2 to T3 ( $p=.004, p<.001$, and $p<.001$, respectively), significantly transmuting to non-caseness ( $p<.001$ for T1 to T2; $p=.025$ for T2 to T3). Between T1 and T2, decreases in cortisol ( $p<.001)$, diastolic blood pressure ( $p<.001)$, and systolic blood pressure $(p<.001)$ were demonstrated. Heart rate fell nonsignificantly between T1 and T2 ( $p=.063)$, but significantly from T1 to T3 ( $p=.048)$. The findings of this study indicate the potential mutability of the psychophysiological illness-prone characteristics of Type D personality.
\end{abstract}

Keywords: Type D personality, cortisol, cardiovascular parameters, havening techniques, clinical trial

Type D personality is indicated by psychophysiological distress and characterized by the interaction between its negative affect (NA) and social inhibition (SI) constituents (Denollet et al., 2006). This personality construct has been associated with disease, somatic complaints (Jellesma, 2008), immunological vulnerability, health status, and the development of psychiatric disorders (Williams et al., 2008). In a systematic review, Mols and Denollet (2010) concluded that Type D is a "vulnerability factor for general psychological distress that affects mental and physical health status and is associated with disease-promoting mechanisms and work-related problems in apparently healthy individuals" (p. 1). Type D personality manifests in approximately $30 \%$ of the population (Horwood et al., 2015), and given the longitudinal health impact of prolonged distress (Olive et al., 2016), the exploration of targeted therapeutic interventions is a worthwhile area of consideration in psychophysiological research to address the adverse impact on health outcomes (Pedersen, Lemos, et al., 2004).

Type D personality is grounded in the theoretical principle that the simultaneous presentation of NA and SI is a determinant of psychophysiological distress, and a risk factor for a range of detrimental health consequences. Mols and Denollet (2009) broadly define NA as "the tendency to experience negative emotions" (p. 66). NA has a demonstrable impact on neuroendocrine underpinnings of disease and a wide variety of physiological concomitants (Herbert \& Cohen, 1993), alongside established links with coronary heart disease (Frasure-Smith et al., 1995). Furthermore, NA is correlated with maladaptive health behaviors, reduced health-protective routines (Dalton \& Hammen, 2018; Pedersen, Van Domburg, et al., 2004), and medication non-adherence (Molloy et al., 2012). The SI constituent 
of Type D is associated with a consistent tendency to "inhibit the expression of emotions and behaviors in social interaction" (Denollet, 2000, p. 256). SI has been associated with a range of adverse health outcomes including reduced heart rate variability (Carpeggiani et al., 2005), increased inflammation (Maes et al., 1998), and reduced treatment adherence (Pereira et al., 2004).

The adverse impact of Type D personality on outcomes in patients with known cardiovascular disease has been widely researched (Pedersen, Lemos, et al., 2004). In a 5 -year follow-up of 541 patients with coronary heart disease, the combined NA and SI score (as a continuous measure) was associated with a significantly higher rate of the combined end-point of cardiac death and acute myocardial infarction after adjustment for age, sex, depressive symptoms, and disease severity (Denollet et al., 2013). This study found a continuum of increased risk with combined NA and SI scores, even among those who did not fulfill Type D caseness cut-off. Importantly, participants with high NA or SI scores alone were not at significantly increased risk. Furthermore, Type D personality is associated with established cardiovascular risk factors. Compare et al. (2014) reported a significant association between Type D and the presence of coronary artery atheromatous plaque detected by computed tomography in 75 participants without a history of coronary heart disease and this remained significant after adjustment for conventional cardiac risk factors as covariates. Hypertension increases vulnerability to myocardial infarction, heart failure, and stroke (Wilson et al., 1998). Therefore, the sustained elevation of blood pressure frequently observed in Type D personality indicates a contributing risk of cardiovascular disease (Denollet, 2005).

Previous psychophysiological research has identified distress-activated cortisol as a mediating physiological antagonist. Van Eck et al. (1996) found that negative affect is related to elevated salivary cortisol secretion during normal daily activities. Chronic distress also results in activation of the sympathetic nervous system leading to hypertension, tachycardia, and activation of the hypothalamic-pituitaryadrenal axis, resulting in hypercortisolemia (Dickerson \& Kemeny, 2004). According to Conrad (2008), the adverse psychophysiological consequences associated with chronic distress predominantly result from cortisol-mediated neurotoxicity in the hippocampus. Accordingly, clinical psychological medicine studies identify elevations in heart rate, blood pressure, and evidence of neuroendocrine stress indices as robust physiological stress biomarkers in stress testing methodology such as the "Trier Social Stress Test" (Kirschbaum et al., 1993). Therefore, when examining the literature in relation to Type D mechanisms, it is imperative to consider the inextricable links with cortisol and blood pressure to prevent "biological disturbances resulting from chronic and uncontrollable stressors" (Herriot et al., 2018, p. 850).

Denollet (2000) maintained that the Type D personality construct is a risk factor for both cardiovascular disease and mental ill health (including post-traumatic stress responses). A number of studies have examined this link. In an examination of the stress responses of $N=151$ prison staff, Kunst et al. (2009) found that Type D personality predisposed individuals to the development of Post-Traumatic Stress Disorder (PTSD). Cho and Kang (2017) found in regression analysis that Type D personality significantly predicted PTSD symptoms. In addition, the symptomatic experience of post-trauma symptoms was demonstrated to be more severe in firefighters with high Type D scores (Orginíska-Bulik \& Langer, 2007). However, Rademaker et al. (2011) in a prospective study of military personnel, found no significant contribution of Type D personality to the prediction of post-deployment trauma symptoms. Although the links between Type D personality and trauma symptoms have yet to be established, it is a developing area of research interest.

Blair (2008) considered the intrinsic physiological functional contributions of distress and asserted that the amygdala is strongly implicated in stimulus-reinforcement learning (particularly where there is maladaptive trauma or distress-oriented cognitive and behavioral patterns). Havening Techniques (HT) are an intervention intended to affect amygdala-based distress cycles by using neurochemical pathway reactivity to alter how responses to trauma and distress are encoded (Ruden, 2011). HT is based on the principle that sensory input can alter brain function by re-establishing neurochemical resilience and reducing chronic distress.

The HT sequencing pattern is comprised of three elements grounded in a theoretical understanding of neurocognitive responses to distress: (1) brief activation of emotional distress, (2) sensory input/distraction, and (3) Havening touch. Extensive research into neurochemical response to massage (Field et al., 2005) has demonstrated the efficacy of touch in decreasing plasma cortisol while raising dopamine and serotonin. Ruden (2018) has proposed that the kinaesthetic component of HT augments serotonin and consequently promotes gamma-aminobutyric acid release, recognized to increase delta wave activity (Ciranna, 2006; Darbari et al., 2005). This process is outlined in the model presenting the HT cortisol distress reduction pathway in Figure 1. Van de Helm et al. (2011) performed functional magnetic resonance imaging (fMRI) and quantitative electroencephalographic (qEEG) studies and found that delta waves during REM (rapid eye movement) sleep depotentiates amygdala activity to neurochemical and emotional reactivity. The researchers in this study suggested that this mechanism of enabling psychological 


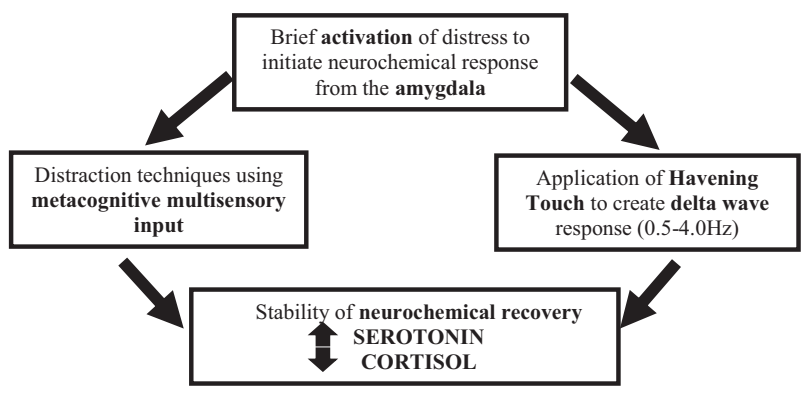

Figure 1. A model presenting the HT cortisol distress reduction pathway.

and physiological recovery is particularly relevant to the REM and delta wave abnormalities associated with traumatic responses. Furthermore, this hippocampal shift to the delta wave state was found to decrease cortisol levels in a study by Van Cauter et al. (2000) which correlated cortisol levels with polysomnographic recordings.

Multi-sensory metacognitive distractions are incorporated in HT in parallel with touch to enable the displacement of distress-oriented cognition and disrupt negatively encoded pathways (Ruden \& Ruden, 2010). The theoretical rationale for this constituent originates from research on memory retrieval mechanisms, indicating that focusing on a distressing memory in parallel with completing a distraction task creates competition for working memory retrieval resources (Morey et al., 2009). Taxing the working memory reduces the distress of the memory and acts as a barrier to memory retrieval (Gunter \& Bodner, 2008), this inhibits outflow of the neurotransmitter glutamate in the basolateral complex which contributes to emotional distress cycles associated with repetitive memory retrieval (Ruden, 2011) and cultivates neurochemical resilience. Gursimran et al. (2015) assessed the efficacy of a single session HT intervention in 27 health workers with a history of anxiety and/or depression related occupational health impairment. Three validated self-assessment questionnaires were employed to assess anxiety, depression, and functional impairment. HT significantly improved all scores at 1 week and further significant improvements were reported 2 months post intervention. The study reports encouraging results, although its limitations included a small sample size and the absence of a control group. However, the evaluation of a treatment grounded in psychophysiological theory offers an opportunity for further examination utilizing an evidence-based framework.

This statistician-blind parallel-group controlled trial was designed to test the hypothesis that HT administered to participants presenting with Type D personality characteristics at baseline (T1) was efficacious in decreasing Type D Scale-14 (DS14) scores 24 hours post intervention (T2). The secondary aims were to evaluate effects of HT on biomarkers (heart rate, blood pressure, and salivary cortisol) at $\mathrm{T} 2$ in a subgroup of participants, and to assess whether early effects on DS14 scores and biomarkers were sustained at T3 (one-month post-treatment).

\section{Methods}

\section{Participants}

Participants were recruited from existing self-referral trauma resolution therapy caseloads of certified HT practitioners in England following practitioner assessment for Type D personality characteristics (based on the predefined criteria established by Denollet, 2005). Following enrolment, participants completed the DS14 questionnaire and were included in this study irrespective of whether DS14 Type D caseness criteria were fulfilled (i.e., score cut-off $\geq 10$ for each domain), as continuum effects were observed in previous research (Denollet et al., 2013), and taxometric analysis research advises against categorical rigidity of the DS14 measure (Ferguson et al., 2009). In addition, inclusion criteria specified a sample of cognizant adults (age 18+ years), exclusion criteria for the biomarker evaluation subgroup included a history of Hepatitis B, tuberculosis or HIV due to clinical laboratory regulations.

The two-domain structure of the DS14 questionnaire (Denollet, 2005), provides one score for NA and the second for SI. Sample size calculations specified NA score to examine physiological effects (as advocated by Coyne \& de Voogd, 2012). Sample size calculations were based on a study that investigated the impact of an 8-week self-referral mindfulness-based stress reduction intervention on Type D personality (Nyklicek, Beugen, \& Denollet, 2013). The reported effect size of the mindfulness-based stress reduction intervention on NA was 0.6. In order to perform sample size estimates, a similar mean baseline score of 16 and $S D=5.2$ was anticipated in this HT intervention trial. One hundred six participants ( $>53$ in each group) are required to identify a reduction in the score of 3 in the treatment versus the waiting list group with $90 \%$ power and $\alpha=.05$. Therefore, this study necessitated the recruitment of a minimum of 55 participants in the waiting list group and 55 in the treatment group to ensure that the minimum sample size calculations were achieved, allowing for a dropout of two participants in each group.

\section{Procedure}

The research design employed a statistician-blind parallel group comparison of HT treatment and waiting list groups. The practitioners were selected because their practices represented varying waiting list timescales for treatment (half 
held a waiting list timescale for treatment engagement greater than 2 months, the other half of less than a week), enabling a natural group allocation process. An initial (nontreatment) assessment was provided to determine the appropriate support needs of each individual. The waiting list group received their initial assessment, and were cognizant of the waiting timescales from the outset Waiting list participants subsequently received treatment following their participation in this study. Informed consent was obtained from all participants. In accordance with clinical trial recommended guidelines, all participants continued to see their health care providers while enrolled on this trial.

The treatment group participants attended an appointment to receive a single session of HT administered by a certified practitioner. The intervention comprised: the brief activation of distress, HT touch applied to the face, arms and palms, and specific distraction techniques. As this research was designed to test the hypothesis that HT was efficacious on the DS14 Type D measure of personality, the DS14 questionnaire was utilized pre-treatment (T1), $24 \mathrm{hr}$ post-treatment (T2), and 1 month later (T3). Participants accessed a Qualtrics questionnaire via either a PC or smartphone in privacy to minimize practitioner bias; the input was designed to take under $15 \mathrm{~min}$. The waiting list group completed the questionnaires at parallel time points without receiving the intervention.

The biomarker evaluation subgroup of the treatment group was assessed for blood pressure (BP), heart rate (HR), and salivary cortisol in parallel with psychometric testing at each timepoint to explore the influence of HT on somatic pathophysiology. Collection of biomarkers was undertaken by 11 practitioners who had a nursing or medical professional qualification, prior to completing the questionnaire in privacy.

\section{Measures}

To address the research aims, the use of the brief, psychometrically sound and validated Type D Scale-14 (DS14) measure of Type D personality (Denollet, 2005) was identified as appropriate following extensive literature review: The DS14 measure of Type D personality is a 14item psychometric questionnaire that utilizes a 5-point Likert scale, each item weighted from 0 to 4 . The DS14 incorporates two 7-item subscales measuring the NA and SI constituents of Type D. A score of $\geq 10$ on both domains confirms the presence of Type D caseness (i.e., meeting the criteria for Type D). The DS14 demonstrates evidence of internal consistency $(\alpha=.88 / .86 ; N=3,678)$, and testretest reliability $(r=.72 / .82)$ over a period of 3 months (Denollet, 2005). Emons et al. (2007) used item response theory to evaluate the psychometric properties of the DS14 and found that in patients with cardiovascular disease it is a valid measure. A secure online survey software program (Qualtrics) was used to collect and collate data. Embedded in this survey was a participant ID entry system, information and informed consent form (tick box), a brief demographic questionnaire (containing the sociodemographic variables of age and gender), the DS14 questionnaire, a blood pressure reporting system (for those in the biomarker subgroup), and a debriefing section including a referral link to further support.

Specific biomarker selection replicated earlier research by Habra, Linden, Anderson, and Weinberg (2003) which examined the relationship between Type D personality and indices of cardiovascular health. At all three data timepoints; cardiovascular parameters and salivette samples were taken following $30 \mathrm{~min}$ of rest in a sitting position. Salivary cortisol levels were reported to be elevated in Type D personality (Whitehead et al., 2007); contemporary studies adopt a preference for salivary cortisol sampling versus venepuncture methodology to eliminate the procedural stress variable associated with blood tests (Dickerson \& Kemeny, 2004). Salivette samples were taken at identical times of day (late morning to avoid circadian variation), participants were instructed to avoid caffeine and eating for at least 90 min prior to saliva sampling as this abstention period is necessary because abrupt rises in cortisol levels have been found in this period following food intake (Toda et al., 2004). Samples were frozen $\left(-20{ }^{\circ} \mathrm{C}\right)$ and transferred to the laboratory on dry ice. To ensure anonymity, the laboratory implemented a data barcode system matched to participant IDs. Samples were held at the medical laboratory and stored purely for the purposes of this trial in accordance with the Human Tissues Act (Acts of Parliament, 2004) research practice guidelines (paragraph 117-123). Clinical laboratory excellence guidelines and legislation were adhered to. HR, systolic blood pressure (SBP), and diastolic blood pressure (DBP) were measured (using an Omron HEM-722C device, Omron Company, Kyoto, Japan). A standard arm cuff was placed on the participant's non-dominant arm, and participants were advised to remain still during cuff inflation for accurate measurement. Blood pressure measurements were given in units of millimeters of mercury $(\mathrm{mmHg})$.

\section{Data Analysis}

Raw data for statistical analysis were recorded within the Qualtrics database and subsequently analyzed using SPSS for Windows version 23 (IBM Corporation, NY) by a researcher blinded to treatment allocation (in order to minimize potential for research bias). The primary end-point was change in DS14 score between T1 and T2, the 
secondary aims were to evaluate whether these effects persisted one-month later (at T3), and the effect on psychobiological variables. Prior to statistical analysis, a Kolmogorov-Smirnov test was performed, and data were normally distributed therefore parametric analyses were conducted. Distributions of categorical variables were compared using $\chi^{2}$ tests. A mixed analysis of variance (ANOVA) was undertaken to evaluate the interaction between time and treatment allocation on psychometric scores. Where a significant interaction was observed, unpaired $t$-tests were performed to compare changes between groups in NA, SI, and total DS14 scores between T1 and T2, T1 and T3, and $\mathrm{T} 2$ and $\mathrm{T} 3$ in the treatment and waiting list group. While the co-primary end points were the DS14 scores and their constituents (NA and SI), analysis was also undertaken for changes in Type D caseness (i.e., total DS14 score of at least 10) in order to provide a context regarding the clinical significance of the change in scores.

For analysis of the impact of HT on biomarker variables: Salivary cortisol, systolic blood pressure (SBP), diastolic blood pressure (DBP), heart rate (HR), and mean blood pressure $(\mathrm{MBP})$ calculated by $(2 \times \mathrm{DBP}+\mathrm{SBP}) / 3$ were compared at T1, T2, and T3. A one-way repeated-measures analysis of variance (RM-ANOVA) was performed, with Bonferroni correction, using time as the independent variable and each physiological parameter as dependent variables. Where a significant interaction was observed, a paired $t$-test was performed to compare these biomarkers between $\mathrm{T} 1$ and $\mathrm{T} 2, \mathrm{~T} 1$ and $\mathrm{T} 3$, and $\mathrm{T} 2$ and $\mathrm{T} 3$. The relationship between changes in DS14 score and changes in biomarkers was assessed using Pearson's correlation coefficient.

Figure 2 presents a consort flow diagram detailing the research phases and participant engagement in this parallel-group controlled trial. The primary analysis was an intention-to-treat approach where missing data were imputed using the last-observation-carried-forward method in accordance with standard practice for an intervention trial. However, a per-protocol approach where data from participants who withdrew and were excluded from initial analysis is also presented in order to address the extent to which the Type D personality construct is potentially modifiable.

\section{Results}

\section{Baseline Characteristics}

A total of 68 and 57 participants were recruited into the treatment and waiting list groups, respectively, although 3 (treatment) and 1 (waiting list) were lost to follow-up. At treatment endpoint (T3) there were 125 participants
(56 waiting list, 25 psychometrics only treatment subgroup, 40 psychobiological, and psychometric evaluation treatment subgroup). This was less than the anticipated dropout rate of $5 \%$, therefore sample size calculations were exceeded across both groups. The distribution of men and women in the treatment and waiting list groups was well-matched (treatment group 42 of 68 vs. waiting list 30 of 57 female; $\chi^{2}=0.72, p=.397$ ). Participants' age data were collected as a categorical variable representing 5 bands. Age distribution (\%) did not differ significantly between treatment and waitlist groups $\left(\chi^{2}=4.4, p=.351\right)$; age band: $18-24$ (5.9 vs. 15.8 ), $25-34$ (13.2 vs. 7 ), $35-44$ (29.4 vs. 24.6$), 45-54$ (20.6 vs. 19.3 ), 55 and over (30.9 vs. 33.8$)$.

In the treatment group, 59 of $68(87 \%)$ participants met the DS14 criteria for type D caseness versus 54 of 57 (95\%) in the waiting list group $\left(\chi^{2}=3, p=.229\right)$. As shown in Table 1, total DS14 scores did not differ significantly at baseline (T1) between treatment and waiting list groups, $t(111)=-0.94, p=.348$, Glass's $\Delta=0.13$. NA and SI scores were also similar at baseline between groups, $t(108)=1.25$, $p=.215$; and $t(110)=-0.97, p=.334$, Glass's $\Delta=0.14$, respectively. DS14 scores were comparable in the biomarker and psychometric only treatment subgroups at baseline ( $M=40.3, S D=11.9$ and $M=41.4, S D=11.3$, respectively $)$ $t(66)=0.4, p=.691$, Glass's $\Delta=0.09$.

\section{Efficacy of Havening Techniques on Psychometric Questionnaire Scores and Type D Caseness}

As demonstrated in Figure 3 and Table 5 Type D caseness remained stable across all three timepoints in the waiting list participants $(p=.887)$. In the treatment group, caseness fell from T1 to T2 $\left(\chi^{2}=60, p<.001\right)$ and further from T2 to T3 $\left(\chi^{2}=7.4, p=.025\right)$. Two non-Type D participants at $\mathrm{T} 1$ met caseness at T2, and 44 who met Type D caseness at T1 no longer met it at T2, and 7 who met caseness at $\mathrm{T} 2$ no longer met it at $\mathrm{T} 3$, and 2 who did not meet it at $\mathrm{T} 2$ met caseness at $\mathrm{T} 3$.

The results of the DS14 questionnaire in the treatment and waiting list groups at $\mathrm{T} 1, \mathrm{~T} 2$, and $\mathrm{T} 3$ are presented in Table 1. While the DS14 data remained stable over time in the waiting list participants, substantial decreases in both NA and SI occurred post intervention. To assess whether these changes were significant, a mixed ANOVA was performed using time and treatment allocation as the independent variables; highly significant time versus treatment interactions were observed for NA, SI, and total DS14 scores (Table 2). As significant time-treatment interactions were identified, post-hoc unpaired $t$-tests were performed to compare changes in psychometric score data in 


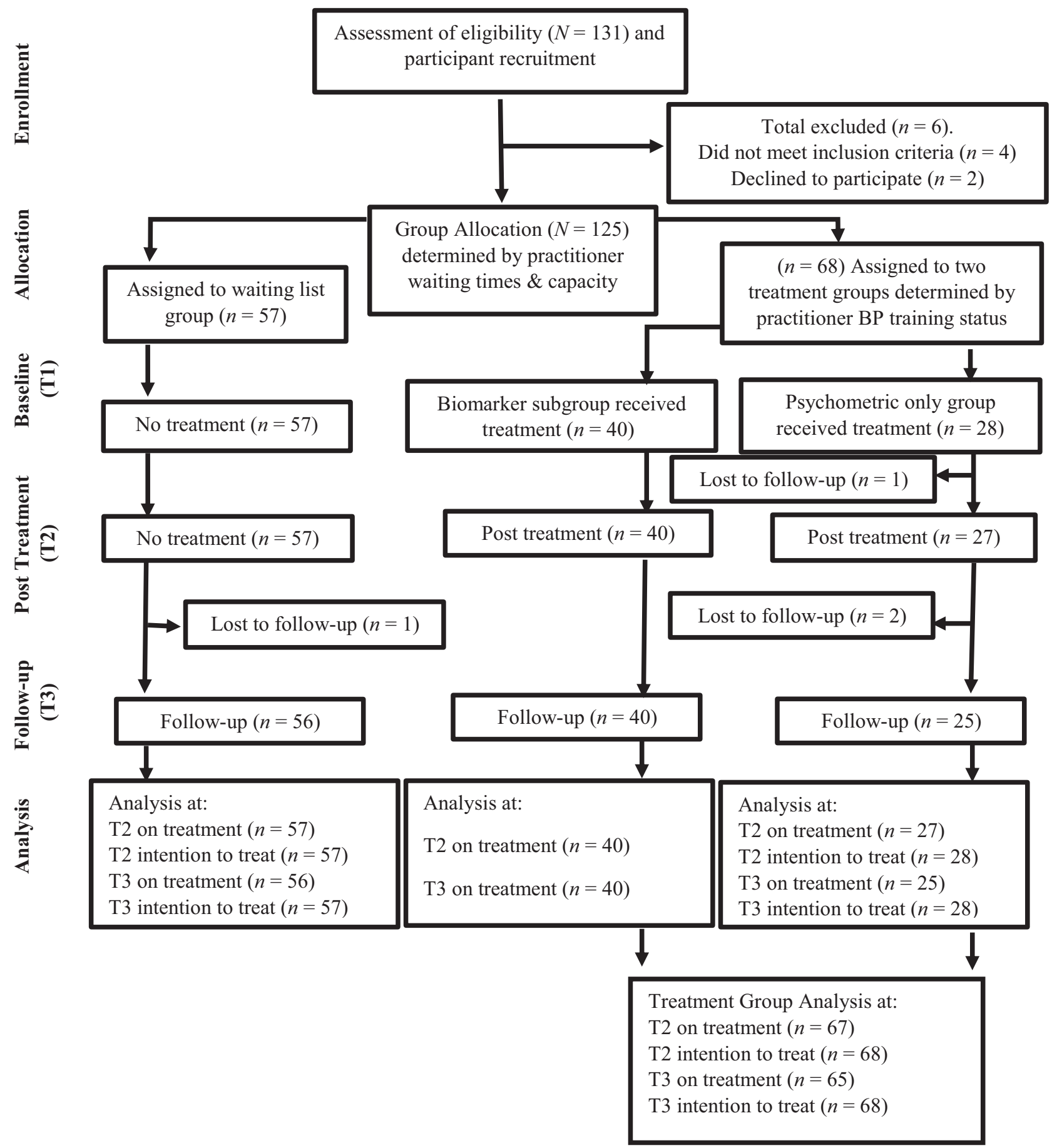

Figure 2. A CONSORT flow diagram of the research phases from enrolment to analysis. Group Allocation Descriptions: Waiting list: (participants who remained on a waiting list until their completion in this study); Treatment Group (all participants receiving the treatment intervention); biomarker subgroup (treatment group participants whose outcomes were assessed by psychometric and stress biomarker analysis); Psychometric Only Subgroup (treatment group participants whose outcomes were assessed only by psychometric analysis).

treatment versus waiting list groups between $\mathrm{T} 1$ and $\mathrm{T} 2$, $\mathrm{T} 1$ and $\mathrm{T} 3$, and $\mathrm{T} 2$ and $\mathrm{T} 3$. The results are summarized in Table 3. Effect sizes were calculated as Glass's $\Delta$ to account for different variances between the groups. Compared to the waiting list group, the treatment group demonstrated statistically significant reductions in NA and SI scores and in total DS14 scores between T1 and T2, and further reductions between $\mathrm{T} 2$ and $\mathrm{T} 3$. 
Table 1. Mean psychometric scores at T1, T2, and T3 in the treatment and waiting list groups

\begin{tabular}{|c|c|c|c|c|}
\hline Analysis & $\begin{array}{l}\text { Psychometric } \\
\text { scores }\end{array}$ & $\begin{array}{c}\text { Time point } \\
\text { (T) }\end{array}$ & $\begin{array}{l}\text { Treatment group } \\
\quad(n=68) M(S D)\end{array}$ & $\begin{array}{l}\text { Waiting list group } \\
(n=57) M(S D)\end{array}$ \\
\hline \multirow[t]{9}{*}{ Per-protocol } & \multirow[t]{3}{*}{ NA } & $\mathrm{T} 1$ & $21.7(5.5)$ & $20.8(2.8)$ \\
\hline & & $\mathrm{T} 2$ & $9.2(5.9)$ & $21.0(3.4)$ \\
\hline & & T3 & $6.9(5.6)$ & $20.7(3.0)$ \\
\hline & \multirow[t]{3}{*}{ SI } & $\mathrm{T} 1$ & $19.3(8.1)$ & $18.2(4.7)$ \\
\hline & & T2 & $8.8(3.6)$ & $19.1(4.4)$ \\
\hline & & T3 & $7.1(3.8)$ & $20.3(5.0)$ \\
\hline & \multirow[t]{3}{*}{ Total DS14 } & $\mathrm{T} 1$ & $40.7(11.5)$ & $39.2(6.7)$ \\
\hline & & $\mathrm{T} 2$ & $18.0(8.3)$ & $40.2(6.7)$ \\
\hline & & T3 & $14.0(8.1)$ & $40.8(6.6)$ \\
\hline \multirow[t]{9}{*}{ Intention-to-treat } & \multirow[t]{3}{*}{ NA } & $\mathrm{T} 1$ & $21.7(5.5)$ & $20.8(2.8)$ \\
\hline & & T2 & $10.0(6.3)$ & $21.0(3.4)$ \\
\hline & & Т3 & $7.9(5.7)$ & $20.3(3.6)$ \\
\hline & \multirow[t]{3}{*}{ SI } & $\mathrm{T} 1$ & $19.3(8.1)$ & $18.2(4.7)$ \\
\hline & & $\mathrm{T} 2$ & $9.6(4.7)$ & $19.1(4.4)$ \\
\hline & & T3 & $7.8(4.6)$ & $20.0(5.3)$ \\
\hline & \multirow[t]{3}{*}{ Total DS14 } & T1 & $40.7(11.5)$ & $39.2(6.7)$ \\
\hline & & T2 & $19.2(9.8)$ & $40.2(6.7)$ \\
\hline & & Т3 & $15.0(9.2)$ & $40.1(7.7)$ \\
\hline
\end{tabular}

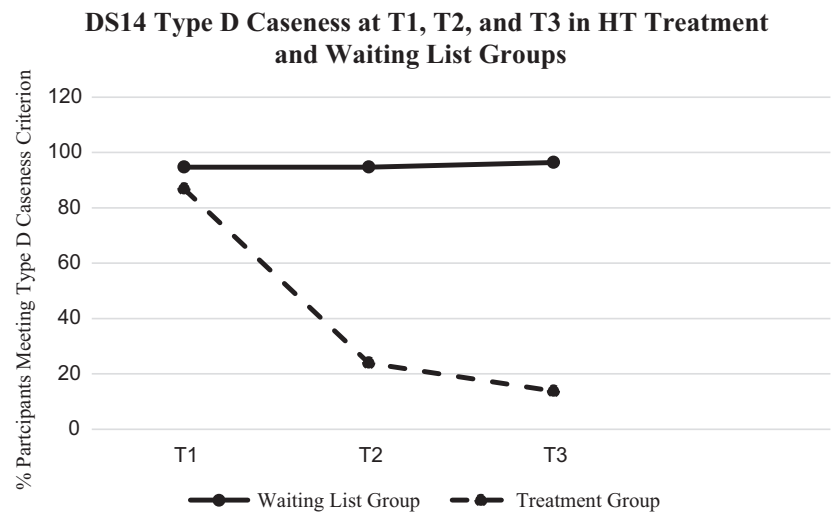

Figure 3. Percentage of participants meeting DS14 cut-off for Type D caseness at T1, T2, and T3 in treatment and waiting list groups.

\section{Efficacy of Havening Techniques in the Biomarker Evaluation Subgroup}

The demographic characteristics of the biomarker measurement subgroup were comparable to those of the treatment and waiting list groups: for biomarker subgroup versus total treatment group, age: $\chi^{2}=3.1(p=.54)$ and gender: $\chi^{2}=0.48(p=.55)$; and for biomarker subgroup versus waiting list, age: $\chi^{2}=7.5(p=.11)$ and gender: $\chi^{2}=0.053$ $(p=.839)$. Furthermore, baseline and post-intervention DS14 scores in the biomarker subgroup were comparable to those observed in the total treatment group (Table 1).
The evaluation analyses are per-protocol only, as no participants in the subgroup were lost to follow-up. Figure 4 illustrates biomarker outcomes at $\mathrm{T} 1, \mathrm{~T} 2$, and $\mathrm{T} 3$.

Reductions in biomarker parameters were evident following HT (Figure 4). SBP, in $\mathrm{mmHg}$, decreased from T1 $(M=131.6, S D=21.1)$ to $\mathrm{T} 2(M=126.2, S D=17.9)$, declining further from T2 to T3 $(M=122.9, S D=12.8)$. DBP, in $\mathrm{mmHg}$, reduced from $\mathrm{T} 1(M=86.1, S D=15.0)$ to $\mathrm{T} 2$ $(M=81.7, S D=12.7)$, declining further from $\mathrm{T} 2$ to $\mathrm{T} 3$ $(M=80.1, S D=10.7)$. MBP, in $\mathrm{mmHg}$, also decreased from $\mathrm{T} 1(M=101.2, S D=16.3)$ to $\mathrm{T} 2(M=96.5, S D=13.7)$, lowering further from T2 to T3 $(M=94.4, S D=10.1)$. Heart rate, in bpm, reduced from $\mathrm{T} 1(M=74.6, S D=11.9)$ to $\mathrm{T} 2(M=72.9, S D=11.6)$, decreasing marginally from $\mathrm{T} 2$ to T3 $(M=72.2, S D=11.1)$. Salivary cortisol, in $\mathrm{ng} / \mathrm{mL}$, decreased from $\mathrm{T} 1(M=5.3, S D=3.5)$ to $\mathrm{T} 2(M=4.2, S D$ $=2.8)$, declining further from $\mathrm{T} 2$ to $\mathrm{T} 3(M=3.7, S D=$ 3.3). To assess whether the differences were significant, a one-way RM-ANOVA was performed on biomarkers with time as the independent variable. Mauchly's test indicated that the assumption of sphericity was violated for these biomarkers $(p<.001$ in all cases); therefore, the reported $p$-values and degrees of freedom are adjusted using Greenhouse-Geisser correction. Highly significant interactions were found between time and systolic BP, diastolic BP, mean BP, and cortisol: $F(1,1.5)=16.7, p<.001 ; F(1,1.3)$ $=17.1, p<.001 ; F(1,1.3)=23.9, p<.001 ;$ and $F(1,1.5)=$ $40.1, p<.001$, respectively. There was a modest interaction between time and heart rate, $F(1,1.4)=3.9, p=.024$. 
Table 2. Mixed ANOVA on psychometric variables - time and treatment allocation as independent variables

\begin{tabular}{|c|c|c|c|c|c|c|c|c|c|}
\hline \multicolumn{5}{|c|}{ Per-protocol analysis } & \multicolumn{5}{|c|}{ Intention-to-treat analysis } \\
\hline $\begin{array}{l}\text { Psychometric } \\
\text { measures }\end{array}$ & Variable & F statistic & $d f$ & $P$ & $\begin{array}{l}\text { Psychometric } \\
\text { measures }\end{array}$ & Variable & F statistic & $d f$ & $P$ \\
\hline \multirow[t]{3}{*}{ NA } & Time & 144.0 & 2 & $<.001$ & & Time & 130.0 & 2 & $<.001$ \\
\hline & Treatment & 107.0 & 1 & $<.001$ & NA & Treatment & 114.0 & 1 & $<.001$ \\
\hline & Time $\times$ Treatment & 133.0 & 2 & $<.001$ & & Time $\times$ Treatment & 116.0 & 2 & $<.001$ \\
\hline \multirow[t]{3}{*}{ SI } & Time & 52.9 & 2 & $<.001$ & & Time & 51.5 & 2 & $<.001$ \\
\hline & Treatment & 99.1 & 1 & $<.001$ & SI & Treatment & 75.7 & 1 & $<.001$ \\
\hline & Time $\times$ Treatment & 88.6 & 2 & $<.001$ & & Time $\times$ Treatment & 84.7 & 2 & $<.001$ \\
\hline \multirow[t]{3}{*}{ Total DS14 } & Time & 121.0 & 2 & $<.001$ & Total DS14 & Time & 107.0 & 2 & $<.001$ \\
\hline & Treatment & 213.0 & 1 & $<.001$ & & Treatment & 140.0 & 1 & $<.001$ \\
\hline & Time $\times$ Treatment & 140.0 & 2 & $<.001$ & & Time $\times$ Treatment & 122.0 & 2 & $<.001$ \\
\hline
\end{tabular}

Note. Results are divided into per-protocol and intention-to-treat analyses.

Table 3. Change in psychometric scores in treatment and control groups between time points

\begin{tabular}{|c|c|c|c|c|c|c|}
\hline Analysis & Time points & Measures & $\begin{array}{l}\text { Treatment group } \\
M(S D)\end{array}$ & $\begin{array}{l}\text { Waiting list group } \\
M(S D)\end{array}$ & $p$ & $\begin{array}{l}\text { Glass's } \Delta \\
\text { effect size }\end{array}$ \\
\hline \multirow[t]{9}{*}{ Per-protocol } & \multirow[t]{3}{*}{ T1 vs. T2 } & NA (DS14) & $-12.3(7.4)$ & $-0.1(3.0)$ & $<.001$ & 4.0 \\
\hline & & SI (DS14) & $-10.0(8.4)$ & $0.6(3.2)$ & $<.001$ & 3.3 \\
\hline & & Combined DS14 & $-22.2(14.4)$ & $0.5(5.6)$ & $<.001$ & 6.0 \\
\hline & \multirow[t]{3}{*}{ T1 vs. T3 } & NA (DS14) & $-15.0(7.6)$ & $-0.4(2.3)$ & $<.001$ & 6.2 \\
\hline & & SI (DS14) & $-11.9(8.8)$ & $1.9(3.5)$ & $<.001$ & 3.9 \\
\hline & & Combined DS14 & $-26.9(14.9)$ & $1.1(4.2)$ & $<.001$ & 4.8 \\
\hline & \multirow[t]{3}{*}{ T2 vs. T3 } & NA (DS14) & $-2.5(4.4)$ & $-0.4(2.3)$ & .001 & 1.0 \\
\hline & & SI (DS14) & $-2.0(3.9)$ & $1.2(3.9)$ & $<.001$ & 1.1 \\
\hline & & Combined DS14 & $-4.5(7.2)$ & $0.5(4.6)$ & $<.001$ & 1.4 \\
\hline \multirow[t]{9}{*}{ Intention-to-treat } & \multirow[t]{3}{*}{ T1 vs. T2 } & NA (DS14) & $-11.7(7.5)$ & $-0.1(3.0)$ & $<.001$ & 3.8 \\
\hline & & SI (DS14) & $-9.8(8.5)$ & $0.6(3.2)$ & $<.001$ & 3.2 \\
\hline & & Combined DS14 & $-21.5(14.7)$ & $0.5(5.6)$ & $<.001$ & 3.9 \\
\hline & \multirow[t]{3}{*}{ T1 vs. T3 } & NA (DS14) & $-13.9(8.0)$ & $-0.4(2.3)$ & $<.001$ & 5.7 \\
\hline & & SI (DS14) & $-11.6(8.9)$ & $1.9(3.5)$ & $<.001$ & 3.8 \\
\hline & & Combined DS14 & $-25.7(15.6)$ & $1.1(4.2)$ & $<.001$ & 6.3 \\
\hline & \multirow[t]{3}{*}{ T2 vs. T3 } & NA (DS14) & $-2.2(4.2)$ & $-0.4(2.3)$ & .004 & 0.8 \\
\hline & & SI (DS14) & $-1.8(3.8)$ & $1.2(3.9)$ & $<.001$ & 0.8 \\
\hline & & Combined DS14 & $-4.2(7.1)$ & $0.5(4.6)$ & $<.001$ & 1.0 \\
\hline
\end{tabular}

Significant interactions were identified between time and the biomarkers, therefore post hoc paired $t$-tests were performed to assess changes between $\mathrm{T} 1$ and $\mathrm{T} 2, \mathrm{~T} 1$ and $\mathrm{T} 3$, and $\mathrm{T} 2$ and $\mathrm{T} 3$. As summarized in Table 4, significant reductions in systolic, diastolic and mean blood pressures were found between each time interval. Heart rate fell significantly between $\mathrm{T} 1$ and $\mathrm{T} 3$ but there was no significant difference between $\mathrm{T} 1$ and $\mathrm{T} 2$ and $\mathrm{T} 2$ and $\mathrm{T} 3$. Salivary cortisol fell significantly between $\mathrm{T} 1$ and $\mathrm{T} 2$, and between $\mathrm{T} 2$ and $\mathrm{T} 3$.

The correlations between DS14 scores and biomarker changes from T1 to T3 was assessed by Pearson's correlations as follows: SBP $r(38)=.453, p=.003$; for DBP $r(38)$ $=.225, p=.163$; for MBP $r(38)=.371, p=.018$; for HR $r(38)=-.012, p=.94$; and for cortisol $r(38)=.237, p=.14$.

\section{Discussion}

The results of this study confirmed the hypothesis that NA, SI and composite DS14 scores would be reduced 24-hourspost-HT. The effect sizes for these changes were substantial, and considerably greater than the 0.6 effect size assumed for the change in the primary end point for the sample size calculation. Furthermore, Type D caseness fell from $86.8 \%$ at $\mathrm{T} 1$ to $34.7 \%$ at $\mathrm{T} 2$ ( $24 \mathrm{hr}$ post-treatment), whereas, in the waiting list group, caseness was identical at T1 and T2 (94.7\%). The efficacy on DS14 scores was paralleled by beneficial effects on stress-related biomarkers that are important in mediating cardiovascular disease risk, with the biomarker evaluation treatment subgroup demonstrating significant reductions in blood pressure and 


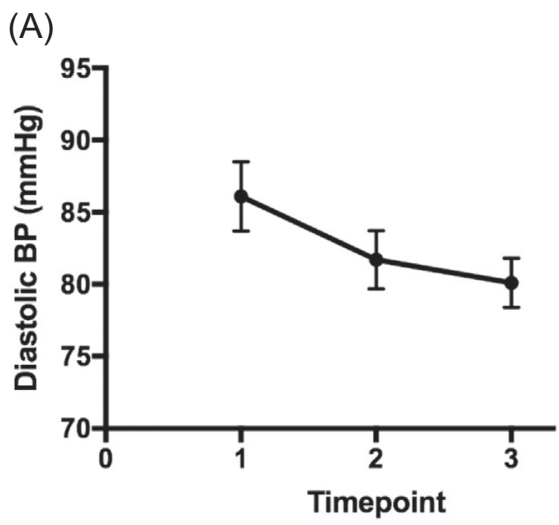

(C)

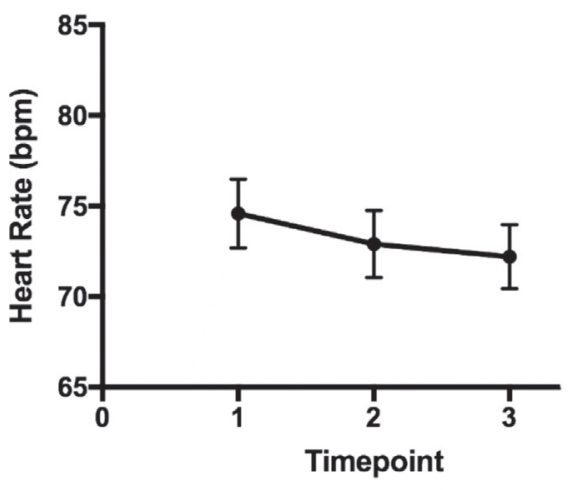

(B)

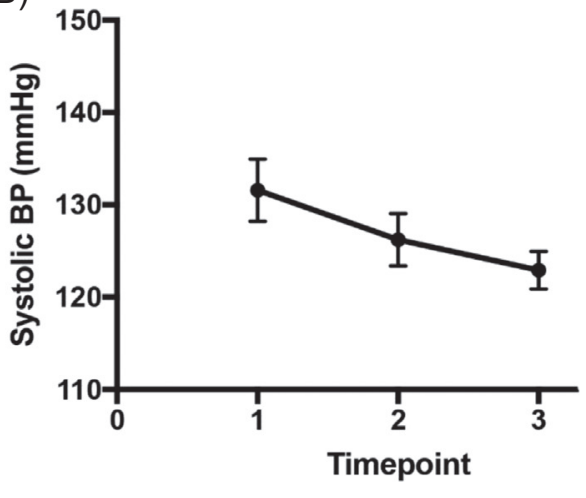

(D)

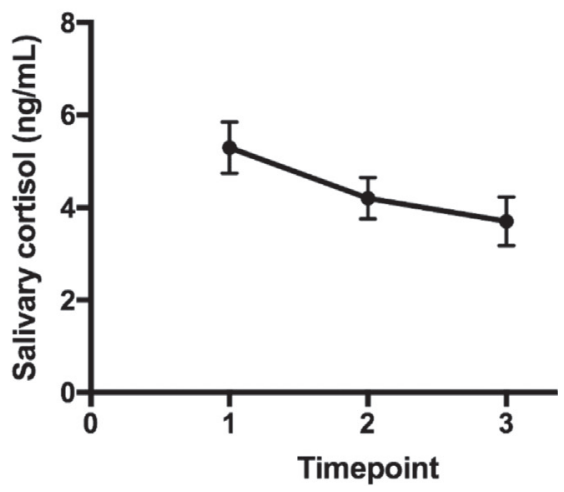

\begin{tabular}{|ll|}
\hline \multicolumn{2}{|l|}{ Effects of HT intervention on Biomarkers } \\
T-test $p$-values for DBP & T-test $p$-values for SBP \\
T2 vs T1 $-p<.001$ & T2 vs T1 $-p<.001$ \\
T3 vs T1 $-p<.001$ & T3 vs T $-p<.001$ \\
T3 vs T2 $-p=.048$ & T3 vs T2 $-p=.016$ \\
& \\
T-test $p$-values for HR & T-test $p$-values for Cortisol \\
T2 vs T1 $-p=.063$ & T2 vs T1 $-p<.001$ \\
T3 vs T1 $-p=.033$ & T3 vs T1 $-p<.001$ \\
T3 vs T2 $-p=.253$ & T3 vs T2 $-p=.003$ \\
\hline
\end{tabular}

Figure 4. Line graphs of biomarker outcomes (mean scores including standard error bars). (A) Diastolic blood pressure; (B) Systolic Blood Pressure; (C) Heart Rate; (D) Salivary Cortisol.

salivary cortisol, and modest reductions in heart rate. There were further improvements in DS14 scores and in biomarkers between T2 (24 hr post-treatment) and T3 (one-month follow-up). In contrast, no significant variation in scores was observed in the waiting list group. There were moderate significant correlations between changes in DS14 scores between $\mathrm{T} 1$ and $\mathrm{T} 3$, and changes in SBP and MBP.

Type D caseness cut-off was met in $94.7 \%$ of the waiting list group $(M=39.2)$ and $86.8 \%$ of the treatment group $(M=40.7)$ at baseline, and most participants were markedly above caseness threshold DS14 scores. The high scores may be explained by the findings of Deane and Chamberlain (2007) who examined the predictors of help-seeking and found that although only a small proportion of people seek professional psychological support; increased distress predicted a higher likelihood of treatment being sought. Despite the stability of Type D in the absence of intervention, significant beneficial effects of an 8-week mindfulness intervention on DS14 scores have previously been demonstrated (Nyklicek, Beugen, \& Denollet, 2013), indicating that it may be a personality construct amenable to intervention. The results in the current HT intervention trial of 
Table 4. Results from post hoc paired t-tests in the biomarker subgroup

\begin{tabular}{llll}
\hline Biomarkers & \multicolumn{1}{c}{ T1 vs. T2 } & \multicolumn{1}{c}{ T1 vs. T3 } & T2 vs. T3 \\
\hline Systolic BP & 131.6 vs. 126.2, $p<.001$ & 131.6 vs. 122.9, $p<.001$ & 126.2 vs. $122.9, p=.016$ \\
Giastolic BP & Glass's $\Delta=.25$ & Glass's $\Delta=.41$ & Glass's $\Delta=.18$ \\
& 86.1 vs. $81.7, p<.001$ & 86.1 vs. $80.1, p<.001$ & 81.7 vs. 80.1, $p=.048$ \\
Mean BP & Glass's $\Delta=.29$ & Glass's $\Delta=.35$ & Glass's $\Delta=.07$ \\
& 101.2 vs. $96.5, p<.001$ & 101.2 vs. $94.4, p<.001$ & 96.5 vs. $94.4, p=.009$ \\
Heart rate & Glass's $\Delta=.29$ & Glass's $\Delta=.42$ & Glass's $\Delta=.16$ \\
& 74.6 vs. $72.9, p=.063$ & 74.6 vs. $72.2, p=.033$ & 72.9 vs. $72.2, p=.253$ \\
Salivary cortisol & Glass's $\Delta=.14$ & Glass's $\Delta=.2$ & Glass's $\Delta=.06$ \\
& 5.3 vs. $4.2, p<.001$ & 5.3 vs. $3.7, p<.001$ & 4.2 vs. 3.7, $p=.003$ \\
\hline
\end{tabular}

Table 5. The effect of HT intervention on the proportion of participants fulfilling Type D caseness criteria

\begin{tabular}{lll}
\hline Timepoint & Treatment group & Waiting list group \\
\hline T1 & $n=59$ of 68 & $n=54$ of 57 \\
T2 & $n=16$ of $67^{*}$ & $n=54$ of 57 \\
T3 & $n=9$ of $65^{+}$ & $n=54$ of 56 \\
\hline
\end{tabular}

Note. ${ }^{*} \chi^{2}=60 ; p<.001$ (T2 vs. T1 in treatment group); ${ }^{+} \chi^{2}=7.4 ; p=.025$ (T3 vs. T2 in treatment group).

significant improvement in scores reinforce the observations of Nyklicek, Beugen and Denollet (2013) and support the theory that interventions may be efficacious in decreasing illness propensity and transmuting expression of caseness in this population. The rapidity of effect of HT observed $24 \mathrm{hr}$ after a single intervention is a striking, and perhaps surprising feature of our observations. These results reinforce the challenge posed by the findings of the mindfulness study to the stable personality trait assumptions of Type D personality. Indeed, Roberts and Mroczek (2008) presented evidence that personality traitchange was responsive to life experiences in adulthood, therefore interventions may present comparable causal mechanisms for trait-change.

This study has several limitations. In this study, the effect of HT on biomarker variables was assessed by repeated measures ANOVA. While this is a standard statistical technique in studies such as this, the heterogeneity of time points (i.e., $24 \mathrm{hr}$ and $1 \mathrm{month}$ ) represents a potential limitation, because correlation between data points tends to be highest between adjacent time points. Further, treatment of the participants by the same practitioners may have led to a clustering effect. More detailed analyses in future studies should use marginal or mixed models. Participants in this study were "help-seeking" as they were recruited as a self-referral waiting list cohort for a trauma-resolution therapy. Wilson et al. (2005) asserted that there is a behavior-change intention inherent in help-seeking, this was supported by Zartaloudi and Madianos (2010) who found that people who underutilize self-referral services were less likely to respond to intervention. The addition of a supportive therapist offers positive relational depth, this alone may positively impact on the SI variable by "the capacity of social support to buffer the negative effects of stress" (Wiesmaierova et al., 2019, p. 1), indeed psychophysiological benefits may also be observed as positive social experiences are associated with lower mortality and improved health (Berkman \& Leonard Syme, 1979). A placebo or best-available-therapy control group would address whether this had any impact on stress biomarker or DS14 results and may yield valuable data that achieve doubleblinding as participants were cognizant of their specific treatment allocation in this study.

Single point biomarker measures were explored in this study under standardized conditions (identical times of day, following $30 \mathrm{~min}$ of rest). The biomarker component in this study was undertaken as a pilot. More detailed data would be provided by the inclusion of ambulatory BP measurement. There is inconsistency in the literature regarding the cortisol awakening response in Type D. In survivors of acute coronary syndrome, salivary cortisol was not associated with severity, but cortisol awakening response was positively (and independently) associated with Type D personality (Whitehead et al., 2007). However, recently Smith et al. (2018) demonstrated that there was no significant relationship between basal cortisol levels and Type D personality. Participants in this study were seeking treatment for trauma resolution, and their salivary cortisol levels were elevated compared to local reference values. Pacella et al. (2014) found that treatment of PTSD in females, but not males, was associated with normalization of the cortisol awakening response. Accordingly, on balance, in a definitive study cortisol awakening response may be superior to a single cortisol measurement. The results of this trial indicate that participants' stress biomarkers were reduced following the HT intervention. However, stress biomarkers were measured in resting state, and it would be interesting to examine whether similar effects may be replicated in an experimental stress protocol. 
As the selected measures demonstrated significant improvements, future research recommendations include the examination of a broader range of Type D and stress measures to test the sensitivity of the DS14, the inclusion of diagnostic analysis, and inclusion of a biomarker control group in order to exclude the possible effect of time and repeated assessment on biomarker improvement. Moreover, this clinical trial presents data demonstrating the effect of $\mathrm{HT}$ on heart rate and $\mathrm{BP}$, however, heart rate variability (HRV) analysis provides valuable information regarding baroreflex sensitivity, cardiac parasympathetic and sympathetic tone. As SI has been associated with reduced HRV (Carpeggiani et al., 2005), the use of HRV analysis from electrocardiogram recordings may be useful in future research.

Participants were recruited in this study following practitioner assessment based on the predefined characteristics of Type D personality established by Denollet (2005), a small minority of the identified participants $(5 \%$ of the waiting list group and $13 \%$ of the treatment group) did not meet the DS14 caseness cut-off for Type D personality. Therefore, future research may consider DS14 criteria-based recruitment methods exclusively. Furthermore, the addition of a long-term follow-up period to assess sustained longitudinal efficacy would be advantageous. Although this study included a robust sample size with significant findings, further research is recommended to ensure confident generalizability.

Nevertheless, Type D personality is not a diagnosis, and there are no associated clinical practice guidelines, despite evidence that this construct is associated with impaired well-being and is receptive to intervention (Mols \& Denollet, 2010). Pedersen, Lemos, et al. (2004) argued that the extent to which Type D personality may respond to treatment interventions needs to be examined to assess the potential to decrease the risk of cardiovascular disease. Therefore, the DS14 and stress biomarker measures may have a role in the development and evaluation of evidencebased health-protective interventions that consider the impact on somatic pathophysiology in parallel with psychological well-being.

Historically, the primary focus of psychological medicine research was the "demonstration that psychological influences could alter physiological processes in a way that significantly contributed to pathogenesis" (Friedman \& Benson, 1994, p. 143) and that the clinical approach to disease and health may be positively affected by psychological intervention. Recent research by Ruden (2018) submits that HT endeavors to harness responses to stimuli (particularly electroceutical delta waves) to enable psychophysiological resilience through endogenous reparation. However, these theoretically-grounded conclusions underpinning HT require further investigation in future research using qEEG methodology. Nonetheless, hypothetically, the stimulation of the brain's intrinsic potential (by activating intra-corporally) propounds an intervention with no known side-effects. In conclusion, the marked effect of the intervention on DS14 scores and on caseness, and the psychobiological parameters associated with Type D personality indicates the potential malleability of the personality construct and indicates scope to further explore personalityoriented health promotion and illness prevention interventions from a psychophysiological perspective.

\section{References}

Acts of Parliament. (2004). Human Tissue Act. Crown Publication. http://www.legislation.gov.uk/ukpga/2004/30/pdfs/ukpga_ 20040030_en.pdf

Berkman, L. F., \& Leonard Syme, S. (1979). Social networks, host resistance, and mortality: A nine-year follow-up of Alameda county residents. American Journal of Epidemiology, 109(2), 186-204. https://doi.org/10.1093/oxfordjournals.a-je.a112674

Blair, R. (2008). The amygdala and ventromedial prefrontal cortex: Functional contributions and dysfunction in psychopathy. Philosophical Transactions of the Royal Society B: Biological Sciences, 363(1503), 2557-2565. https://doi.org/10.1098/ rstb.2008.0027

Carpeggiani, C., Emdin, M., Bonaguidi, F., Landi, P., Michelassi, C., Trivella, M., Macerata, A., \& L'Abbate, A. (2005). Personality traits and heart rate variability predict long-term cardiac mortality after myocardial infarction. European Heart Journal, 26(6), 1612-1617. https://doi.org/10.1093/eurheartj/ehi252

Cho, G-J., \& Kang, J. (2017). Type D personality and posttraumatic stress disorder symptoms among intensive care unit nurses: The mediating effect of resilience. PLoS One, 12(4), Article e0175067. https://doi.org/10.1371/journal.pone. 0175067

Ciranna, L. (2006). Serotonin as a modulator of glutamate- and GABA-mediated neurotransmission: Implications in physiological functions and pathology. Current Neuropharmacology, 4(2), 101-114. https://doi.org/10.2174/157015906776359540

Compare, A., Mommersteeg, P. M., Faletra, F., Grossi, E., Pasotti, E., Moccetti, T., \& Auricchio, A. (2014). Personality traits, cardiac risk factors, and their association with presence and severity of coronary artery plaque in people with no history of cardiovascular disease. Journal of Cardiovascular Medicine, 15(5), 423-430. https://doi.org/10.2459/jcm.0b013e328365cd8c

Conrad, C. D. (2008). Chronic stress-induced hippocampal vulnerability: The glucocorticoid vulnerability hypothesis. Neuroscience Review, 19(6), 395-411. https://doi.org/10.1515/REVNEURO. 2008.19.6.395

Coyne, J. C., \& de Voogd, J. N. (2012). Are we witnessing the decline effect in the type $\mathrm{D}$ personality literature? What can be learned? Journal of Psychosomatic Research, 73(6), 401-407. https://doi.org/10.1016/j.jpsychores.2012.09.016

Dalton, E. D., \& Hammen, C. L. (2018). Independent and relative effects of stress, depressive symptoms, and affect on college students' daily health behaviors. Journal of Behavioral Medicine, 41(6), 863-874. https://doi.org/10.1007/s10865018-9945-4

Darbari, F. P., Melvin, J. J., Piatt, J. H., Adirim, T. A., \& Kothare, S. $V$ (2005). Intrathecal baclofen overdose followed by withdrawal: Clinical and EEG features. Pediatric Neurology, 33(5), 373-377. https://doi.org/10.1016/j.pediatrneurol.2005.05.017 
Deane, F. P., \& Chamberlain, K. (2007). Treatment fearfulness and distress as predictors of professional psychological helpseeking. British Journal of Guidance and Counselling, 22(2), 207-217. https://doi.org/10.1080/03069889408260315

Denollet, J. (2000). Type D personality: A potential risk factor refined. Journal of Psychosomatic Research, 49(4), 255-266. https://doi.org/10.1016/50022-3999(00)00177-X

Denollet, J. (2005). DS14: Standard assessment of negative affectivity, social inhibition, and Type D personality. Psychosomatic Medicine, 67, 89-97. https://doi.org/10.1097/01.psy. 0000149256.81953 .49

Denollet, J., Pedersen, S. S., Ong, A. T., Erdman, R. A., Serruys, P. W., \& van Domburg, R. T. (2006). Social inhibition modulates the effect of negative emotions on cardiac prognosis following percutaneous coronary intervention in the drug eluting stent era. European Heart Journal, 27(2), 171-177. https://doi.org/ 10.1093/eurheartj/ehi616

Denollet, J., Pedersen, S. S., Vrints, C. J., \& Conraads, V. (2013). Predictive value of social inhibition and negative affectivity for cardiovascular events and mortality in patients with coronary artery disease: The type D personality construct. Psychosomatic Medicine, 75(9), 873-881. https://doi.org/10.1097/PSY. 0000000000000001

Dickerson, S. S., \& Kemeny, M. E. (2004). Acute stressors and cortisol responses: A theoretical integration and synthesis of laboratory research. Psychological Bulletin, 130(3), 355-391. https://doi.org/10.1037/0033-2909.130.3.355

Emons, W. H., Meijer, R. R., \& Denollet, J. (2007). Negative affectivity and social inhibition in cardiovascular disease: Evaluating type-D personality and its assessment using item response theory. Journal of Psychosomatic Research, 63, 27-39. https:// doi.org/10.1016/j.jpsychores.2007.03.010

Ferguson, E., Williams, L., O'Connor, R., Howard, S., Hughes, B. M., Johnston, D. W., Allan, J. L., O'Connor, D. B., Lewis, C. A., Grealy, M. A., \& O'Carroll, R. (2009). A taxometric analysis of Type-D personality. Psychosomatic Medicine, 71(9), 981-986. https://doi.org/10.1097/PSY.06013e3181bd888b

Field, T., Hernandez-Reif, M., \& Diego, M. (2005). Cortisol decreases and serotonin and dopamine increases following massage therapy. International Journal of Neuroscience, 115, 13971413. https://doi.org/10.1080/002074505909564590956459

Frasure-Smith, N., Lesperance, F., \& Talajic, M. (1995). The impact of negative emotions on prognosis following myocardial infarction: Is it more than depression? Health Psychology, 14, 388-398. https://doi.org/10.1037/0278-6133.14.5.388

Friedman, R., \& Benson, H. (1994). Behavioral medicine: A retrospective and a look forward. Journal of Behavioral Medicine, 19(4), 143-144. https://doi.org/10.1080/08964289.1994.9935184

Gunter, R. W., \& Bodner, G. E. (2008). How eye movements affect unpleasant memories: Support for a working-memory account. Behaviour Research and Therapy, 46(8), 913-931. https://doi. org/10.1016/j.brat.2008.04.006

Gursimran, T., Tom, D., Gould, M., McKenna, P., \& Greenberg, N. (2015). Impact of a single-session of Havening. Health Science Journal, 9(5:1), 1-5.

Habra, M. E., Linden, W., Anderson, J. C., \& Weinberg, J. (2003). Type $\mathrm{D}$ personality is related to cardiovascular and neuroendocrine reactivity to acute stress. Journal of Psychosomatic Research, 55(3), 235-245. https://doi.org/10.1016/s0022-3999 (02)00553-6

Herbert, T. B., \& Cohen, S. (1993). Depression and immunity: A meta-analytic review. Psychological Bulletin, 113(3), 472-486. https://doi.org/10.1037/0033-2909.113.3.472

Herriot, H., Wrosch, C., \& Gouin, J. P. (2018). Self-compassion, chronic age-related stressors, and diurnal cortisol secretion in older adulthood. Journal of Behavioral Medicine, 41(6), 850862. https://doi.org/10.1007/s10865-018-9943-6

Horwood, S., Chamravi, D., \& Tooley, G. (2015). Examining the prevalence of Type-D personality in an Australian population. Australian Psychologist, 50, 212-218. https://doi.org/10.1111/ ap.12089

Jellesma, F. C. (2008). Health in young people: Social inhibition and negative affect and their relationship with self-reported somatic complaints. Journal of Developmental and Behavioural Paediatrics, 29(2), 94-100. https://doi.org/10.1097/DBP.0613e31815f24el

Kirschbaum, C., Pirke, K. M., \& Hellhammer, D. H. (1993). The "Trier Social Stress Test" - a tool for investigating psychobiological stress response in a laboratory setting. Neuropsychobiology, 28(1-2), 76-81. https://doi.org/10.1159/000119004

Kunst, M. J., Bogaerts, S., \& Winkel, F. (2009). Peer and inmate aggression, Type D personality and post-traumatic stress among Dutch prison workers. Stress and Health, 25(5), 387395. https://doi.org/10.1002/smi.1247

Maes, M., Sona, C., Lin, A., De Jongh, R., Van Gastel, A., Kenis, G., Bosmans, E., De Meester, I., Benoy, I., Neels, H., Demedts, P., Janca, A., Scharpe, S., \& Smith, R. (1998). The effects of psychological stress on humans: Increased production of proinflammatory cytokines and a Th1-like response in stressinduced anxiety. Cytokine, 10(4), 313-318. https://doi.org/ 10.1006/cyto.1997.0290

Molloy, G. J., Randall, G., Wikman, A., Perkins-Porras, L., MesserliBürgy, N., \& Steptoe, A. (2012). Type D personality, self efficacy, and medication adherence following an acute coronary syndrome. Psychosomatic Medicine, 74(1), 100-106. https://doi. org/10.1097/PSY.06013e31823a5b2f

Mols, F., \& Denollet, J. (2009). Type D personality among noncardiovascular patient populations: A systematic review. General Hospital Psychiatry, 32(1), 66-72. https://doi.org/ 10.1016/j.genhosppsych.2009.09.010

Mols, F., \& Denollet, J. (2010). Type D personality in the general population: A systematic review of health status, mechanisms of disease, and work-related problems. Health and Quality of Life Outcomes, 8(9), 1-10. https://doi.org/10.1186/1477-7525-8-9

Morey, R. A., Dolcos, F., Petty, C. M., Cooper, D. A., Hayes, J. P., LaBar, K. S., \& McCarthy, G. (2009). The role of trauma-related distractors on neural systems for working memory and emotion processing in posttraumatic stress disorder. Journal of Psychiatric Research, 43(8), 809-817. https://doi.org/10.1016/ j.jpsychires.2008.10.014

Nyklicek, I., Beugen, S., \& Denollet, J. (2013). Effects of mindfulness-based stress reduction on distressed (Type D) personality traits: A randomised controlled trial. Journal of Behavioural Medicine, 36(4), 361-370. https://doi.org/10.1007/s10865012-9431-3

Olive, L. S., Telford, R. M., Byrne, D. G., Abhayarratna, W. P., \& Telford, R. D. (2016). Psychological distress leads to reduced physical activity and fitness in children: The Australian longitudinal LOOK study. Journal of Behavioral Medicine, 39(4), 587598. https://doi.org/10.1007/s10865-016-9723-0

Orgińiska-Bulik, N., \& Langer, I. (2007). Type D personality, coping with stress, and intensity of PTSD symptoms in firefighters [Abstract]. Medycyna Pracy, 58(4), 307-316. PMID: 18041200

Pacella, M. L., Feeny, N., Zoellner, L., \& Delahanty, D. L. (2014). The impact of PTSD treatment on the cortisol awakening response. Depression and Anxiety, 31(10), 862-869. https://doi. org/10.1002/da.22298

Pedersen, S. S., Lemos, P. A., van Vooren, P. R., Liu, T. K., Daemen, J., Erdman, R. A., \& Smits, P. C. (2004). Type-D personality predicts death or myocardial infarction after bare metal stent or sirolimus-eluting stent implementation. Journal 
of the American College of Cardiology, 44(5), 997-1001. https:// doi.org/10.1016/j.jacc.2004.05.064

Pedersen, S. S., van Domburg, R. T., Theuns, D. A., Jordaens, L., \& Erdman, R. A. (2004). Type D personality is associated with increased anxiety and depressive symptoms in patients with an implantable cardioverter defribulator and their partners. Psychosomatic Medicine, 66(5), 714-719. https://doi.org/10.1097/ 01.psy. 0000132874.52202 .21

Pereira, D., Antoni, M., Danielson, A., Simon, T., Efantis-Potter, J., \& O'Sullivan, M. (2004). Inhibited interpersonal coping style predicts poorer adherence to scheduled clinic visits in human immunodeficiency virus infected women at risk for cervical cancer. Annals of Behavioral Medicine, 28(3), 195-202. https:// doi.org/10.1207/s15324796abm2803_8

Rademaker, A. R., van Zuiden, M., Vermetten, E., \& Geuze, E. (2011). Type D personality and the development of PTSD symptoms: A prospective study. Journal of Abnormal Psychology, 120(2), 299-307. https://doi.org/10.1037/a0021806

Roberts, B. W., \& Mroczek, D. (2008). Personality trait change in adulthood. Current Directions in Psychological Science, 17(1), 31-35. https://doi.org/10.1111/j.1467-8721.2008.00543.x

Ruden, R. A. (2011). When the past is always present: Emotional traumatisation, causes and cures. Routledge.

Ruden, R. A. (2018). Harnessing electroceuticals to treat disorders arising from traumatic stress: Theoretical considerations using a psychosensory model. EXPLORE, 15(3), 222-229. https://doi. org/10.1016/j.explore.2018.05.005

Ruden, S. J., \& Ruden, R. A. (2010). Treating the phobic and anxious dental patient: Introduction to Havening Therapy. Dentistry Today, 177, 1-6. PMID: 20408291

Smith, M. A., Riccalton, V. C., Kelly-Hughes, D. H., Craw, O. A., Allen, S. F., O'Connor, D. B., \& Wetherell, M. A. (2018). The relationship between Type $D$ personality and physical health complaints is mediated by perceived stress and anxiety but not diurnal cortisol secretion. Stress, 21(3), 229-236. https://doi. org/10.1080/10253890.2018.1435637

Toda, M., Morimoto, K., Nagasawa, S., \& Kitamura, K. (2004). Effect of snack eating on sensitive salivary stress markers cortisol and chromogranin A. Environmental Health and Preventative Medicine, 9(1), 27-29. https://doi.org/10.1265/ehpm.9.27

Van Cauter, E., Leproult, R., \& Plat, L. (2000). Age-related changes in slow wave sleep and REM sleep and relationship with growth hormone and cortisol levels in healthy men. Journal of the American Medical Association, 284(7), 861-868. https://doi. org/10.1001/jama.28

Van Eck, M., Berkhof, H., Nicolson, N., \& Sulon, J. (1996). The effects of perceived stress traits, mood states, and stressful daily events on salivary cortisol. Psychosomatic Medicine, 58, 447-458. https://doi.org/10.1097/00006842-199609000-00007

Van de Helm, E., Yao, J., Dutt, S., Rao, V., Saletin, J. M., \& Walker, M. P. (2011). REM sleep depotentiates amygdala activity to previous emotional experiences. Current Biology, 21(23), 20292032. https://doi.org/10.1016/j.cub.2011.10.052

Whitehead, D. L., Perkins-Porras, L., Strike, P. C., Magid, K., \& Steptoe, A. (2007). Cortisol awakening response is elevated in acute coronary syndrome patients with type-D personality. Journal of Psychosomatic Research, 62, 419-425. https://doi. org/10.1016/j.jpsychores.2006.11.005

Wiesmaierova, S., Petrova, D., Arrebola Moreno, A. A., Catena, A., Ramírez Hernández, J. A., \& Garcia-Retamero, R. (2019). Social support buffers the negative effects of stress in cardiac patients: A cross-sectional study with acute coronary syndrome patients. Journal of Behavioral Medicine, 42(3), 469-479. https://doi.org/10.1007/s10865-018-9998-4

Williams, L., O'Connor, R. C., Howard, S., Hughes, B. M., Johnston, D. W., Hay, J. L., O’Connor, D. B., Lewis, C. A., Ferguson, E.,
Sheehy, N., Grealy, M. A., \& O'Carroll, R. E. (2008). Type-D personality mechanisms of effect: The role of health-related behavior and social support. Journal of Psychosomatic Research, 64(1), 63-69. https://doi.org/10.1016/j.jpsychores.2007.06.008

Wilson, P. W., D’Agostino, R. B., Levy, D., Belanger, A. M., Silbershatz, H., \& Kannel, W. B. (1998). Prediction of coronary heart disease using risk factor categories. Circulation, 97(18), 18371847. https://doi.org/10.1161/01.CIR.97.18.1837

Wilson, C. J., Deane, F. P., Ciarrochi, J. V., \& Rickwood, D. (2005) Measuring help-seeking intentions: Properties of the General Help-Seeking Questionnaire. Canadian Journal of Counselling, 39(1), 15-28.

Zartaloudi, A., \& Madianos, M. G. (2010). Mental health treatment fearfulness and help-seeking. Issues in Mental Health Nursing, 31(10), 662-669. https://doi.org/10.3109/01612840.2010.490929

\section{History}

Received April 26, 2020

Revision received May 26, 2020

Accepted July 2, 2020

Published online September 2, 2020

\section{Acknowledgements}

Sincere gratitude to the participants and practitioners who offered their time to support this research, and to Dr Paul McKenna, Professor Michael Frenneaux, Lorraine O'Mullane, Carol Jones, Dr Caroline Limbert, Dr Prem Chandra, Dr Allán Laville, Dr Daniel Lamport, and Professor Philip James for their professional encouragement. Thanks also to Professor Johan Denollet for his permission to use the DS14 measure of Type D personality in this clinical trial.

\section{Conflict of Interests}

Kirsty L. Hodgson, Debbie A. Clayton, William D. Fraser, and Donnie Cameron declare that they have no conflicts of interest. Ronald Ruden, Michael A. Carmi, and Louise H. Carmi declare professional affiliation with Havening Techniques: These authors contributed to the methodology, practitioner recruitment, and manuscript review; conflicts were managed by their exclusion from data collection and analysis.

\section{Publication Ethics}

All procedures followed were in accordance with the ethical standards of the institutional research committee and with the 1964 Helsinki declaration and its later amendments or comparable ethical standards. Informed consent was obtained from all individual participants included in the study.

The clinical trial registration number for this trial is NCT03568591.

\section{Authorship}

Kirsty L. Hodgson is now at the School of Psychology and Clinical Language Sciences, University of Reading, UK. Debbie A. Clayton is now at Cardiff School of Sport and Health Sciences, Cardiff Metropolitan University, UK.

\section{Kirsty L. Hodgson}

c/o Donnie Cameron

Norwich Medical School, Floor 2

Bob Champion Research and Education Building

James Watson Rd

University of East Anglia

Norwich, NR4 7UQ

UK

praxisconsulting@live.co.uk 